\title{
Analysis and Optimization of the Performance of Rear-seat Female Occupant in Frontal Crash Based on Euro-NCAP
}

\author{
Libo CAO ${ }^{1,2, a}$, Feixia LIU ${ }^{1, b}$, Lingbo YAN ${ }^{1, c}$, Zhi Wang ${ }^{2,3, d}$
}

${ }^{1}$ State Key Laboratory of Advanced Design and Manufacture for Vehicle Body, Hunan University, Changsha, China, 410082

${ }^{2}$ State Key Laboratory of Vehicle NVH and Safety Technology, Chongqing, China, 400023

${ }^{3}$ Changan Automotive Engineering Institute, Chongqing, China, 400023

ahdclb@163.com, ${ }^{\text {b }}$ feixia0915@163.com, cmilanylb@hotmail.com, ${ }^{\mathrm{d}}$ wangzhi@changan.com.cn

Keywords: Frontal crash, Rear seat, female occupant, Safety performance optimization.

\begin{abstract}
The purpose of this study was to identify main influencing parameters to the responses of the rear-seat female occupants in frontal crash and enhance the protection for rear-seat female occupants with specific design. The results suggested parameters of the restraint system exerted significant influence on the injury severity of the female occupant in rear seats. After parameter optimization, the neck score, the chest score and the general score of the female dummy based on the assessment protocol for adult occupant protection of Euro-NCAP increased by 3.6\%, 13.3\% and $3.73 \%$, respectively. Protection of female occupants in rear seat could be enhanced by systematically matching the parameters of the restraint system.
\end{abstract}

\section{Introduction}

Rear seat occupants constitute $14 \%$ of all vehicle occupants in passenger cars and LTVs [1]. However, the real world data suggests that the fatality and serious injury risk in frontal crashes is higher in rear seats than for those in frontal seats [2]. At present, most studies of vehicle passive safety at home and abroad concentrate on drivers and front occupants, while there are few studies focused on rear occupant injury in frontal crashes [3]. Euro-NCAP added $50 \mathrm{~km} / \mathrm{h}$ front impact test in 2015 and all the crew, including the driver, front occupant and rear occupant, are female dummies [4]. In this paper, computational simulations of the female dummy in the rear seats were performed based on MADYMO software. The effective factors of the advanced restraint system features on the protection of rear-seat female occupants were analyzed and optimized.

\section{Methods}

Development and validation of the rear-seat female restraint system model. In this study, a MADYMO model was built to represent the rear-seat compartment based on a $50 \mathrm{~km} / \mathrm{h}$ frontal crash test and the geometric data of a domestic car, which mainly included the frontal seat, the rear seat, the floor pan, seat belt model and the Hybrid III 5th percentile ellipsoid Q dummy. In order to simulate the interaction between the dummy and the rear-seat model, the contact algorithm available was only defined [5]. The model predicted and experimentally measured dummy kinematic data in the crash test were shown in Fig. 1. It can be seen that the patterns of the curves were very similar. Good correlations between the simulation and the test were achieved with average CORrelation and Analysis (CORA) correlation scores of $85.32 \%$. 

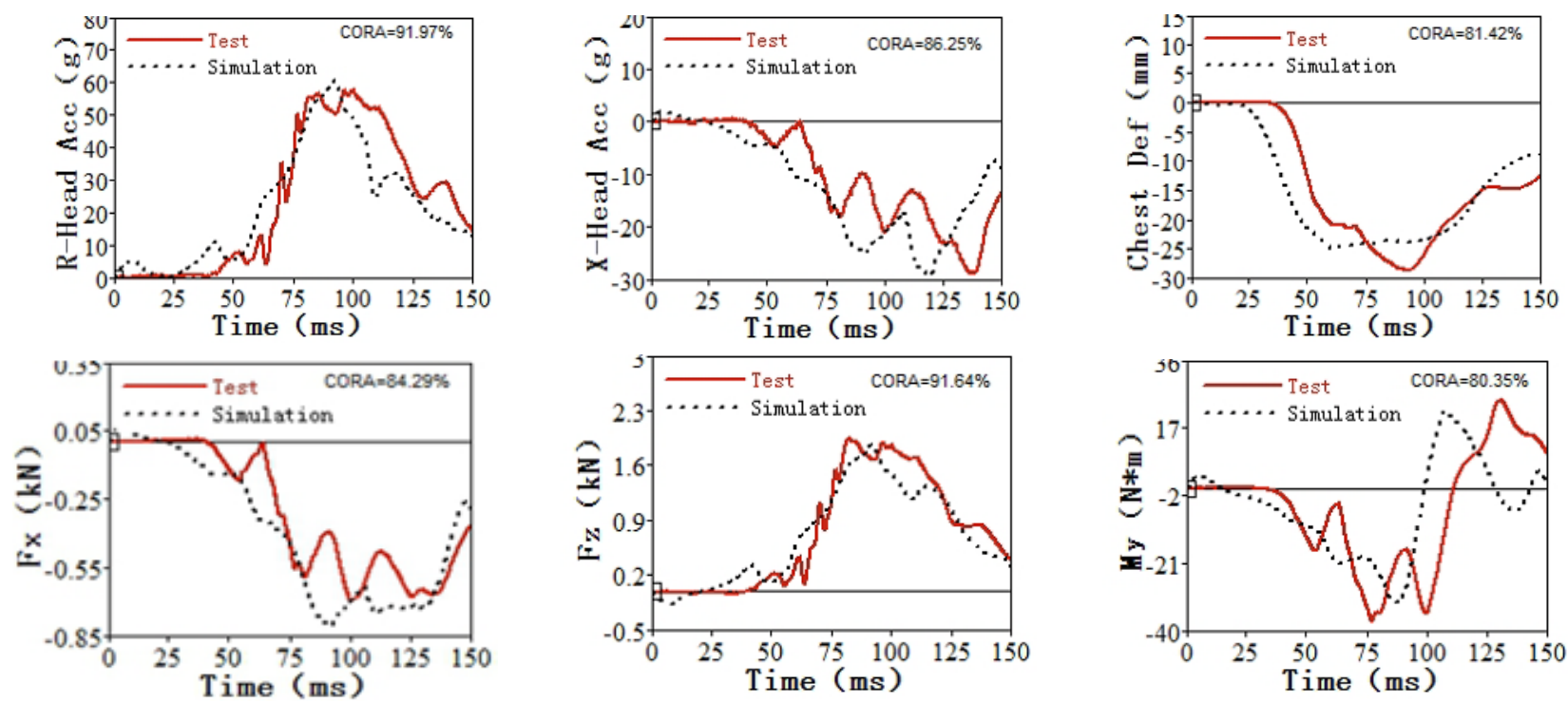

Fig. 1. Comparison of experimental data and simulation results

Sensitivity analysis. After the model had been validated, a parametric sensitivity analysis was performed by MODEFRONTIER to investigate the efficacy of restraint design variables. All of the design variables and the corresponding values range were listed in table 1 . Noticeably, the value range of friction coefficient of the D-ring, and frontal seat backrest angle was continuous. The method of Uniform Latin Square was used to adopt parameters in their scope uniformly. Sensitivity analysis results show in Fig. 2. With the increase of belt load limit, the injury level of all body parts as well as the submarining trend augmented simultaneously. Adversely, both head injury severity and the submarining trend decreased as the seat COF and the seat stiffness increased, respectively. By increasing the TTF of the pretensioner, the neck injury severity showed a moderate increase. In general, belt load limit, seat COF, seat stiffness as well as the TTF of the pretentioner have significant influence on the responses of the rear-seat female occupants.

Table 1 Design variable levels for sensitivity analysis

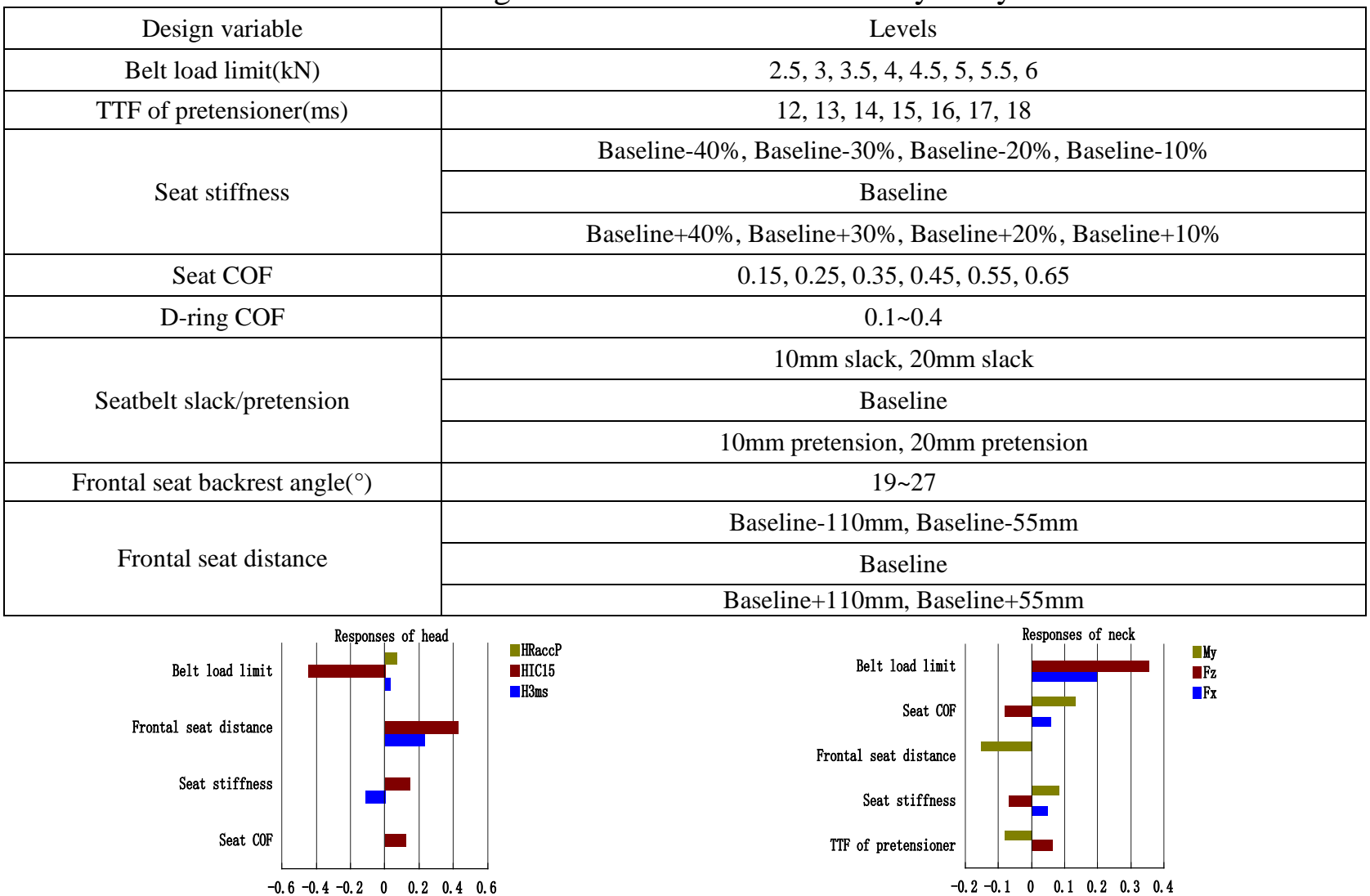




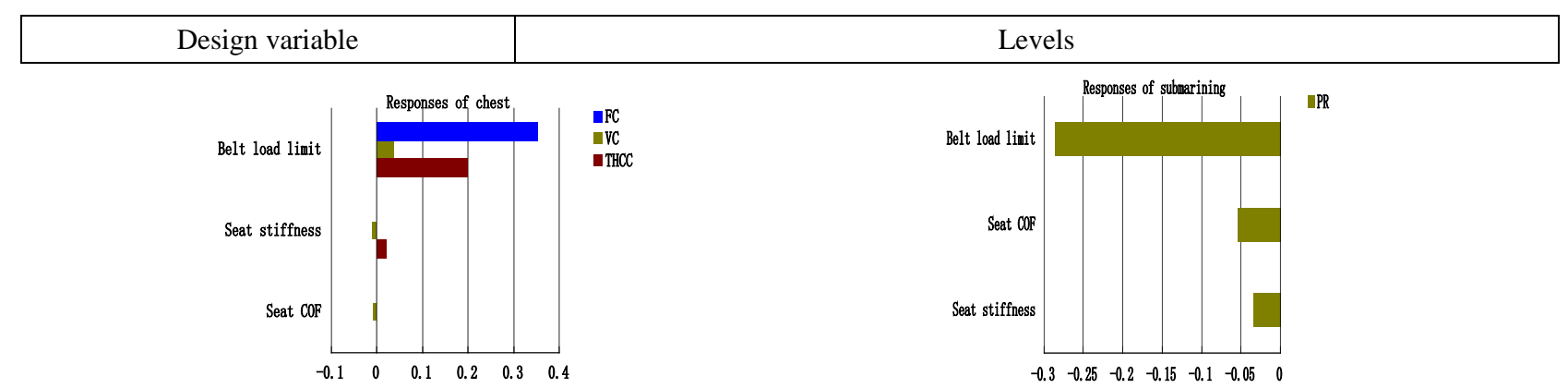

Fig. 2. Sensitivity analysis results $(\mathrm{P}<5 \%$ : $\mathrm{P}$ values are from Kruskal-Wallis one-way ANOVA)

Optimization for the rear-seat female restraint system. In order to improve the protective performance of the rear occupant restraint system, related parameters must be systematically matched. Based on the results of the sensitivity analysis, belt load limit, seat COF, seat stiffness as well as the TTF of the pretensioner were selected as the design variables in order to optimize the rear-seat restraint system with the adoption of the validated rear-seat model integrated with the Hybrid III 5th percentile ellipsoid Q dummy. In this study, the system agent model based on response surface method was used on the occupant restraint system optimization. This method used mathematical model to describe the objective function and constraint function, which fits the system responses through the establishment of approximate function that contains design variables.

Optimization objectives. The score of the rear-seat female occupant based on the assessment protocol for adult occupant protection of Euro-NCAP showed that the chest deformation and the neck tension force caused severe loss of scores. Therefore, those injury parameters should take the priority to be minimized.

Constraint. In the optimization process, the Weighted Injury Criterion (WIC) was used as a constraint condition based on the assessment protocol of adult occupant protection of Euro-NCAP. The definition of WIC ( WIC $\leq 1$ ) was shown as Eq. 1. According to the Euro-NCAP frontal crash test evaluation criteria, a hard contact is assumed, if the peak value of the resultant head acceleration exceeds $80 \mathrm{~g}$ and the shoulder belt force surpasses $6 \mathrm{kN}$ simultaneously, a two point penalty is applied. Both of the situations are not conducive to occupant protection.

$$
W I C=\frac{1}{4}\left[\frac{1}{2}\left(\frac{H I C_{15}}{700}+\frac{H 3 m s}{80}\right)+\frac{1}{4}\left(\frac{F x}{1.95}+\frac{F z}{2.62}+\frac{2 M y}{49}\right)+\frac{1}{2}\left(\frac{T H C C}{42}+V C\right)+\frac{1}{2}\left(\frac{F l+F r}{6.2}\right)\right] .
$$

Response surface. In the experimental design, Uniform Latin method was used to select parameter values within the scope of design variables uniformly. 50 parameter combinations were selected for collision simulations. Simulations were performed automatically with MODE FRONTIER and a table containing the input variables and output variables can be generated by its experiment design (DOE) table data. Kriging Algorithm was used to create the response surface and Genetic Algorithm NSGA-II (Non-dominated Sorting based Algorithm)[6]was used for optimizations. Each optimization analysis included 50 generations and each generation included 50 experiment designs. Each optimization analysis included 2500 simulation tests.

Optimization results. In order to check the reliability of response surface, the optimal design parameters combinations that obtained from optimization were inserted into the validated rear-seat model integrated with the Hybrid III 5th percentile ellipsoid Q dummy to get the calculated injuries. The largest discrepancy between true simulation and virtual simulation was only $7.50 \%$ indicating that response surfaces created above was reliable. The basic model and optimized model scores based on the assessment protocol of adult occupant protection of Euro-NCAP were listed in table 2. As table 2 shown, after optimized, the chest deformation, the neck tension and extension moment were reduced, and the score of the rear-seat female occupant increased by $3.73 \%$. 
Table 2 Assessment of the rear occupant with the optimized restraint system

\begin{tabular}{|c|c|c|c|c|c|c|c|}
\hline \multirow[b]{2}{*}{ Euro-NCAP } & \multirow[b]{2}{*}{ Injury index } & \multicolumn{3}{|c|}{ Basic model } & \multicolumn{3}{|c|}{ Optimized model } \\
\hline & & Injury value & Point & Score & Injury value & Point & Score \\
\hline \multirow{2}{*}{ Head } & $\mathrm{HIC}_{15}$ & 336.44 & 4.00 & \multirow{2}{*}{4} & 206.81 & 4.00 & \multirow{2}{*}{4.00} \\
\hline & H3ms(g) & 58.02 & 4.00 & & 47.85 & 4.00 & \\
\hline \multirow{3}{*}{ Neck } & Shear force $\mathrm{Fx}(\mathrm{kN})$ & 0.80 & 1.00 & \multirow{3}{*}{3.86} & 0.60 & 1.00 & \multirow{3}{*}{4.00} \\
\hline & Tension force $\mathrm{Fz}(\mathrm{kN})$ & 1.83 & 1.86 & & 1.53 & 1.00 & \\
\hline & Extension moment $\mathrm{My}\left(\mathrm{N}^{*} \mathrm{~m}\right)$ & 32.4 & 2.00 & & 26.6 & 2.00 & \\
\hline \multirow{2}{*}{ Chest } & Deformation $(\mathrm{mm})$ & 24.72 & 2.88 & \multirow{2}{*}{2.88} & 22.29 & 3.29 & \multirow{2}{*}{3.29} \\
\hline & $\mathrm{VC}(\mathrm{m} / \mathrm{s})$ & 0.107 & 4.00 & & 0.097 & 4.00 & \\
\hline \multirow{2}{*}{ Femur } & Left compression $(\mathrm{kN})$ & 2.4 & 4.00 & \multirow{2}{*}{4} & 2.475 & 4.00 & \multirow{2}{*}{4.00} \\
\hline & Right compression(kN) & 2.3 & 4.00 & & 2.420 & 4.00 & \\
\hline & eral score & & & 14.74 & & & 15.29 \\
\hline
\end{tabular}

\section{Conclusions}

In this study, a rear-seat female restraint system was developed and validated against the frontal crash test data. Based on the results of the sensitivity analysis, identified main influencing parameters were selected as the design variables in order to optimize the rear-seat restraint system with the adoption of the validated rear-seat female restraint system model. The findings from this study are as follows:

1) The validation results of the rear-seat female occupant restraint system model demonstrated that the model was consistent well with the experimental data.

2) The sensitivity analysis revealed that the main factors that affect the responses of each body region were different: belt load limit exert significant influence on responses of all body parts; The head injuries were influenced by seat COF and seat stiffness; The neck injuries were influenced by the TTF of the pretensioner; Belt load limit, seat COF and the seat stiffness had significant influence on the submarining trend.

3) Protection of female occupants in rear seat could be optimized by systematically matching the belt load limit, seat COF, seat stiffness and the TTF of the pretensioner.

\section{Acknowledgements}

This work was financially supported by State Key laboratory of Vehicle Noise-Vibration-Harshness and Safety Technology fund project (NVHSKL-201415) and Hunan university State Key Laboratory of Advanced Design and Manufacture for Vehicle Body national key experimental independent research topic (51275001).

\section{References}

[1] Foret-Bruno, J-Y., Hartemann, F., Thomas, C, Fayon, A., Tarriere, C., Got, C. (1978) Correlation between thoracic lesions and force values measured at the shoulder belt of 92 belted occupants involved in real accidents. Paper 780892, 22nd Stapp Car Crash Conference, Society of Automotive Engineers, Warrendale, PA.

[2] Kuppa Shashi, et al. Rear Seat Occupant Protection in Frontal Crashes. Paper \#05-212, 19th ESV Conference, Washington, DC.

[3] Qiang Zhan. Save the application of the VIP new technology, make the rear occupant safer. Automotive World. 16-19 (2010),(1). In Chinese.

[4] Michiel van Ratingen, Euro NCAP Roadmap to Implementation 2013 to 2016, 2012 Annual General Meeting of the Automotive Safety Council,Scottsdale, March 2012.

[5] MADYMO Theory Manual. TNO Road-vehicles Research Institute. 209 -251(2005).

[6] Deb, K., et al.: Ieee Transactions on Evolutionary Computation. 182-197(2002)(2). 